\title{
Demanded Dwell Time Reduction Based on Prior RCS Fluctuation Constraint in Target Tracking
}

\author{
Zijian Wu $(\mathbb{D}$, Fei Wang $(\mathbb{D}$, and Jianjiang Zhou \\ College of Electronic and Information Engineering, Nanjing University of Aeronautics and Astronautics, Nanjing 211106, China \\ Correspondence should be addressed to Fei Wang; wangxiaoxian@nuaa.edu.cn
}

Received 4 March 2020; Revised 7 June 2020; Accepted 19 June 2020; Published 25 July 2020

Academic Editor: Everardo Vargas-Rodriguez

Copyright (c) 2020 Zijian Wu et al. This is an open access article distributed under the Creative Commons Attribution License, which permits unrestricted use, distribution, and reproduction in any medium, provided the original work is properly cited.

\begin{abstract}
In the field of airborne radar resource management, one of the most accessible solutions to enhance the power of modern radar system is to save target tracking time as much as possible. During the target tracking process, it is effective to utilize the prior knowledge of the radar cross section (RCS) of the target to save airborne radar resource. Since the target RCS is sensitive to frequency and attitude angle, it is difficult to predict the target RCS accurately. This paper proposes an effective way to save airborne radar time resource in the target tracking process by changing radiation frequency of airborne radar to tolerate certain RCS fluctuation. Firstly, based on the tolerable fluctuation range of the target RCS, this paper designs an effective search algorithm to minimize the frequency set while maximizing the average RCS within the limited fluctuation range. Secondly, the detection probability prediction phase is renewed by taking the RCS fluctuation into account in order to reduce the radar dwell time. Finally, by using interactive multiple model Kalman filter (IMMKF) algorithm, a target tracking procedure with the minimum dwell time prediction method is proposed. Simulation results show that the proposed method is effective. As for target tracking simulation of three different trajectories, the proposed method can save at best $81.35 \%$ more dwell time than the fixed frequency method.
\end{abstract}

\section{Introduction}

As for radar resource management, power and time are two fundamental resources which determine the search space, detection range, and the number of targets that could be tracked simultaneously. Therefore, many methods that can reduce radar radiation power and time are studied, in which minimizing dwell time and maximizing illumination interval are two fundamental ways. The utilization of prior knowledge of target radar cross section (RCS) and then controlling the dwell time of radar radiation can raise the detection probability, thus the illumination interval maximization can be achieved.

As the development of modern computational electromagnetics, taking advantage of the RCS of target becomes accessible to improve radar target detection probability. However, the higher the radar radiation frequency is, the more serious the angular glint will be. Then, the accurate target RCS value is difficult to be determined during the radar target tracking process. During recent years, the study to reveal the relationship between RCS and detection probability has attracted more and more attention. Richards analyzed radar detection probabilities corresponding to chi-square distribution using different Swerling models to describe RCS fluctuation; simulations showed that the radar detection probability would decrease when the RCS fluctuation was dramatic [1]. Using the physical optical principle, Song et al. showed that both Swerling III and IV models could be approximately expressed by Rician distribution, which provided an alternative and reasonable way to model RCS fluctuation distribution of air target [2]. Sun et al. took multiple-input and multiple-output (MIMO) radar into account, assuming that the bistatic RCS of target between an emitter and a receiver of MIMO radar could be predicted in real time to guide the radar to change radiation parameter; the simulations depicted that the tracking performance can be improved if a large number of well-separated antennas were used [3]. Liu et al. compared the influence of RCS fluctuation on radar detection probability and showed that the detection probability of simulated RCS fluctuation value with 
electromagnetic model of target is higher than that with traditional RCS fluctuation models [4]. Väilä et al. proposed to simulate target RCS at first and then took advantage of the predicted entry trajectory to locate early warning radar stations to extend the early warning time [5]. Tan et al. took RCS fluctuation as a target feature and proposed an adaptive radiation waveform design method based on cognitive radar to realize target recognition [6]. Divyalakshmi et al. further proved that the radar detection probability was able to be improved if the target RCS is known [7].

The target RCS and its fluctuation property have been applied to many subjects, such as detection, tracking, and recognition. Radiation waveform design corresponding to the RCS and its fluctuation should be studied in order to increase the detection probability. Bell presented the fundamental information theory for radar waveform design and guided the development of radar waveform design [8]. Recently, Liu et al. analyzed the target RCS property in clutter and proposed an algorithm with the prior knowledge of environment to maximize the target detection performance [9]. Shi et al. assumed that the bistatic RCS fluctuation of the target was limited and proposed an optimal model to balance the low probability of interception performance and the target detection probability by distributed multiple-radar system [10]. Since the wideband radar waveform can be used for imaging and the narrowband radar waveform can be used for detecting, Zhu et al. proposed a technique to design the narrowband waveform with the one-dimensional highresolution range profile of the target [11]. The utilization of target RCS calculation can not only improve radar detection probability but also save radar resources during the target tracking process.

Radar resource management is always an important subject and has been studied for decades. In recent years, Zhang and Tian assumed that the radar power and time resources could be changed based on radar network and the strength of clutter and proposed an optimal resource allocation model to save the total power and time resources of radar network [12]. They extended the power allocation of radar network based on sensor selection algorithm into multiple-target tracking scenario with the constraint of predetermined mutual information [13]. She et al. also showed that the minimum mean-square error could replace the mutual information to select sensors in radar network [14]. As for the complicated electromagnetic environment, Shi et al. introduced game theory to minimize the required radar power [15]. Shi et al. also proposed an optimal model to minimize radar power using orthogonal frequency division multiplex waveform when radar and communication systems were in coexistence [16]. The idea was introduced into the bistatic radar scenarios [17]. Wang et al. proposed that reducing radar dwell time was an important step to prevent radar signal from being tracked by the eavesdropper equipped with a jammer [18].

Based on the previous analysis, RCS fluctuation has been paid more and more attention recently. As far as we know, there are many radar waveform design algorithms utilizing calculated target RCS. The RCS fluctuation models, such as Swerling models, are applied in target tracking process to save radar time resources. However, there has been no paper to study radar signal which could tolerate the calculated target RCS fluctuation, and the existing target tracking algorithms do not take the calculated target RCS fluctuation into account. Since the radar detection probability is proportional to the average RCS, it can be helpful to improve the average RCS by using the appropriate radar waveform carrier frequency.

The purpose of this paper is to reduce radar dwell time during target tracking process using the target RCS prediction method and then improve radar detection probability. The RCS derived from HPEM model is utilized in the process, which in not included in paper [12]. Firstly, by taking the calculated target RCS at all azimuths and all frequency bins into account, a search algorithm is adopted to minimize the corresponding carrier frequency set of radar signal under the constraints of maximizing average RCS and limited RCS fluctuation in continuous azimuths. Secondly, the detection probability prediction phase renewed by different methods, such as optimized method by principle component analysis and optimized method by this paper, is compared. Finally, the prediction step of radar detection probability is applied into the interactive multiple model Kalman filter (IMMKF) algorithm to minimize radar dwell time under the same tracking performance constraint.

\section{Problem Scenario}

An important method to improve radar target detection probability during the target tracking process is to utilize the prior knowledge of target as much as possible. Though the optimal narrowband radiation parameter design of radar carrier frequency based on the knowledge of high resolution range profile (HRRP) of the target is reasonable to improve radar target detection probability in ideal environment, it does not take the observation error into account. The wideband waveform design can be robust to the limited fluctuation of target echo, but it is not suitable for target detection because the signal-to-noise ratio (SNR) is inversely proportional to the bandwidth. The high-precision electromagnetic model (HPEM) of the target is applied to calculate the RCS using high-frequency electromagnetic algorithm, and the database that consists of those RCS results can be set up. A radiation frequency design method based on the target RCS fluctuation constraint is considered; then, the relationship between the RCS fluctuation amplitude and the radar detection probability can be analyzed.

Radar targets in the optical region can be regarded as the combination of multiple scatters so that the target RCS can be calculated with the HPEM model. However, a little movement or a small deformation of the target may lead to random changes of the RCS. Väilä et al. [5] took RCS fluctuation into account and then transform radar detection equation into

$$
P_{\mathrm{r}}=\frac{P_{\mathrm{t}} G_{\mathrm{t}} G_{\mathrm{r}} \lambda^{2} \sigma}{(4 \pi)^{3} L_{\mathrm{s}} L_{\mathrm{f}} R^{4}}
$$




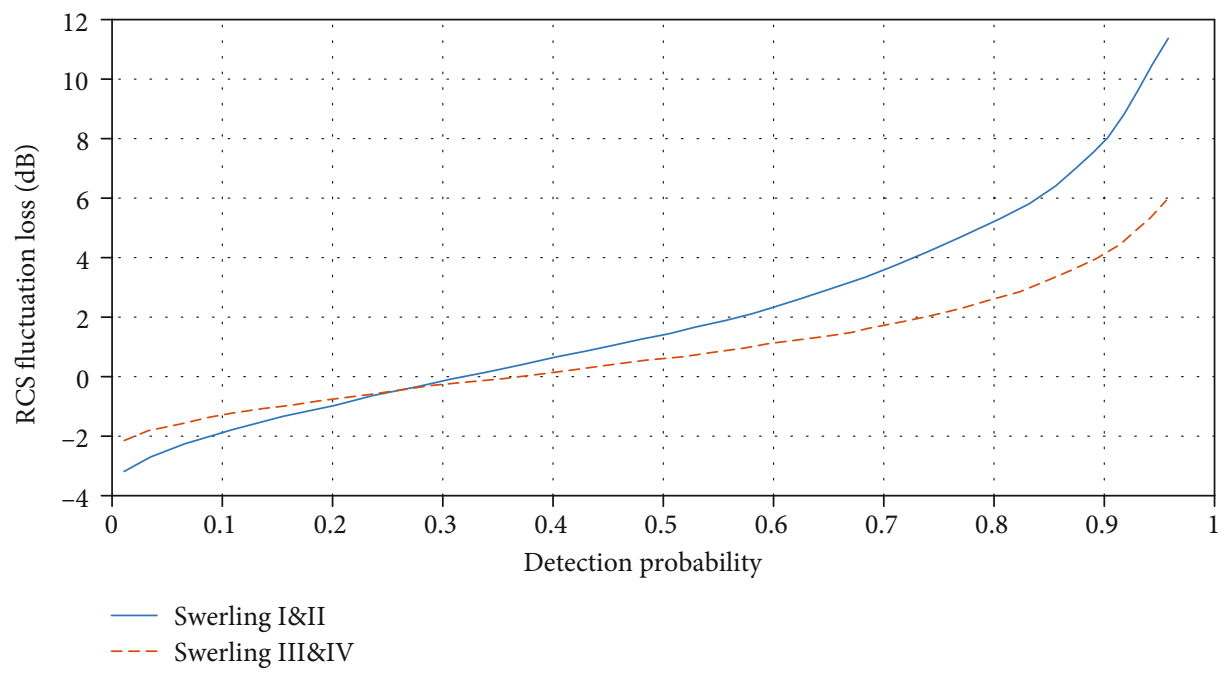

FIGURE 1: The change of detection probability based on RCS fluctuation loss.

where $P_{\mathrm{r}}$ is received echo power; $P_{\mathrm{t}}$ denotes radar transmission power; $G_{\mathrm{t}}$ and $G_{\mathrm{r}}$ denote transmitter antenna gain and receiver antenna gain, respectively; $\lambda$ denotes wavelength; $\sigma$ is target RCS value; $L_{\mathrm{s}}$ and $L_{\mathrm{f}}$ are system loss and target RCS fluctuation loss, respectively; and $R$ is the distance between the radar and target.

Equation (1) can be rewritten as

$$
P_{\mathrm{r}}=K \frac{\lambda^{2} \sigma}{L_{\mathrm{f}} R^{4}}
$$

where $K=\left(P_{\mathrm{t}} G_{\mathrm{t}} G_{\mathrm{r}}\right) /\left((4 \pi)^{3} L_{\mathrm{s}}\right)$, with the definition that $P_{\mathrm{r}}=$ $A^{2} / 2$, and the probability density function $P_{\mathrm{d}}(r)$ of the envelope of $P_{r}$ obeys the generalized Rayleigh distribution

$$
P_{\mathrm{d}}(r)=\frac{r}{\sigma_{0}^{2}} \exp \left(-\frac{r^{2}+A^{2}}{2 \sigma_{0}^{2}}\right) I_{0}\left(\frac{r A}{\sigma_{0}^{2}}\right)
$$

where $r$ is the envelope of the signal plus noise, $\sigma_{0}^{2}$ is the system noise power, $\sigma_{0}^{2}=k F_{\mathrm{n}} T_{0} B_{\mathrm{n}} M, k=1.38 \times 10^{-23}$ is the Boltzmann constant, $F_{\mathrm{n}}$ is the receiver noise, $T_{0}$ is the absolute temperature, $B_{\mathrm{n}}$ denotes receiver bandwidth, $M$ is the modulation factor, and $I_{0}()$ is zero-order modified Bessel function.

Then, there is

$P_{\mathrm{d}}=\int_{\sqrt{2 \sigma_{0}^{2} \ln \left(1 / P_{\mathrm{fa}}\right)}}^{\infty} \frac{r}{\sigma_{0}^{2}} \exp \left(-\frac{r^{2} L_{\mathrm{f}} R^{4}+K \lambda^{2} \sigma}{\sigma_{0}^{2} L_{\mathrm{f}} R^{4}}\right) I_{0}\left(\frac{r \lambda}{\sigma_{0}^{2} R^{2}} \sqrt{2 K \frac{\sigma}{L_{\mathrm{f}}}}\right) d r$

where $P_{\mathrm{fa}}$ is predetermined false alarm probability.

According to Equation (4), the trends of the RCS fluctuation loss versus detection probability are shown in Figure 1 under different Swerling models. Taking Swerling I model as an example [19], in order to maintain the detection probability of 0.9 , an additional $8 \mathrm{~dB}$ SNR (signal-to-noise ratio) increment is needed to compensate the fluctuation loss. This shows that if the fluctuation can be controlled within a limited range, the SNR can be improved, which can help reduce the radar dwell time.

\section{Optimization of Radar Carrier Frequency Set}

Based on the analysis of Section 2, the radar detection performance can be affected by the fluctuation loss of the target RCS, so it can be beneficial to design the matching radar transmission carrier frequency based on the target RCS. If the attitude angle of target is known or predictable, the optimal transmission carrier frequency can be designed by selecting the largest frequency response corresponding to the target.

However, in practical applications, the target RCS is sensitive to attitude angle and the optimal radar carrier frequency design requires too much storage space. Then, this paper proposes a radiation frequency design method based on the target RCS fluctuation constraint. Taking the RCS fluctuation along azimuth angle as an example, the RCS results lower than the preset fluctuation constraint value are classified into one group, and different transmission frequencies for each group are designed to improve the average target detection probability.

Suppose that the target corresponds to the radar transmission frequency $f$ and the RCS with azimuth angles of $j$ and $k$ are $\sigma_{j, f}$ and $\sigma_{k, f}$, respectively, where $k$ is the reference original azimuth of the $i$ th angle span $x_{i}$. The set that can satisfy the target RCS fluctuation loss constraint $\left|\sigma_{j, f}-\sigma_{k, f}\right| \leq \delta$ is

$$
x_{i}=\left\{\mathbf{j}|| \sigma_{j, f}-\sigma_{k, f} \mid \leq \delta\right\}
$$

where $\delta$ is RCS fluctuation loss constraint and

$$
\begin{aligned}
\mathbf{j} & =k+\Delta, \quad \Delta=1,2, \cdots, w_{i}, \\
\mathbf{X} & =\left\{x_{1}, x_{2}, \cdots, x_{M}\right\}, \\
\sum_{i=1}^{M} w_{i} & \geq \text { WID }, \quad i=1,2, \cdots, M,
\end{aligned}
$$


where $w_{i}$ is the width of the $x_{i}$ span, WID represents the interested total azimuth, $M$ represents that WID is partitioned to $M$ span, and $\mathbf{X}$ represents a set that meets the requirement of RCS fluctuation loss constraint.

Assuming the radar transmit frequency corresponding to the set $\mathbf{X}$ consists of a set of transmit frequencies $\mathbf{F}=\left\{f_{1}, f_{2}\right.$, $\left.\cdots, f_{M}\right\}, f_{i}$ is the radar transmission frequency corresponding to the $i$ th azimuth span. Let $|\mathbf{F}|$ denote the total number of elements of the set $\mathbf{F}$; the radiation frequency design algorithm based on the target RCS fluctuation loss constraint is as follows:

(1) Read the target RCS database

(2) Set the RCS fluctuation loss constraint value $\delta$, the interested azimuth angle set, the transmission frequency set, the starting search angle $j_{m}$, and the starting search frequency $f_{n}$

(3) Let the width of the $i$ th angle span be $w_{i}=0$ and $m=1$ (starting from the beginning of the interest angle field)

(4) Compare $\left|\sigma_{j_{m}, f_{n}}-\sigma_{j_{w_{i}-1}, f_{n}}\right|$ and $\delta$ size, $\mid \sigma_{j_{m}, f_{n}}-$ $\sigma_{j_{w_{i}-1}, f_{n}} \mid \leq \delta$. Then, update the search angle to $j_{m-1}$ and $w_{i}+1$, otherwise $w_{i}$

(5) Compare $\left|\sigma_{j_{w_{i}+1}, f_{n}}-\sigma_{j_{m}, f_{n}}\right|$ and $\delta$ size, $\mid \sigma_{j_{m}, f_{n}}-$ $\sigma_{j_{w_{i}+1}, f_{n}} \mid \leq \delta$ Then update the search angle to $j_{m+1}$, and $w_{i}+1$, otherwise $w_{i}$

(6) When $w_{i}$ is unchanged, record $w_{i}$, update the search frequency to $f_{n+1}$, and repeat steps (4) to (6) until all frequency searches are completed

(7) Record the maximum span of the $i$ th angle span $w_{i}$ and the corresponding frequency $f$

(8) Let $j_{m}$ be $j_{w_{i}}$, repeat steps (2) to (8) until all interested azimuth angle set search are completed

\section{Minimum Dwell Time Design Based on RCS Estimation}

4.1. Prediction of Detection Probability. Take the center of the target as the origin of the target coordinate system, then the $X$ -axis along the nose direction, the $Y$-axis is perpendicular to the $X$-axis and is parallel to the left wing of the target, and the $Z$-axis is perpendicular to the $X O Y$ plane. The target attitude angles are the azimuth $\varphi$ and the elevation angle $\theta$ of line of sight of radar in a target body coordinate. Define the azimuth $\varphi$ as the projection of radar line of sight on the XOY plane and set the angles to $\left[-180^{\circ}, 180^{\circ}\right]$. The nose direction is $0^{\circ}$, left wing direction is $90^{\circ}$, and right wing direction is $-90^{\circ}$. Define the elevation angle $\theta$ as the angle between the radar line of sight and $Z$-axis and set the angles to $\left[0^{\circ}, 360^{\circ}\right]$. The view from the top of the target is $0^{\circ}$; the nose cone direction is $90^{\circ}$. The angles are shown in Figure 2.

When a target maneuvers in the radar coordinate system, the radar line of sight changes as time and its attitude vary and the RCS value of the target changes. The transformation

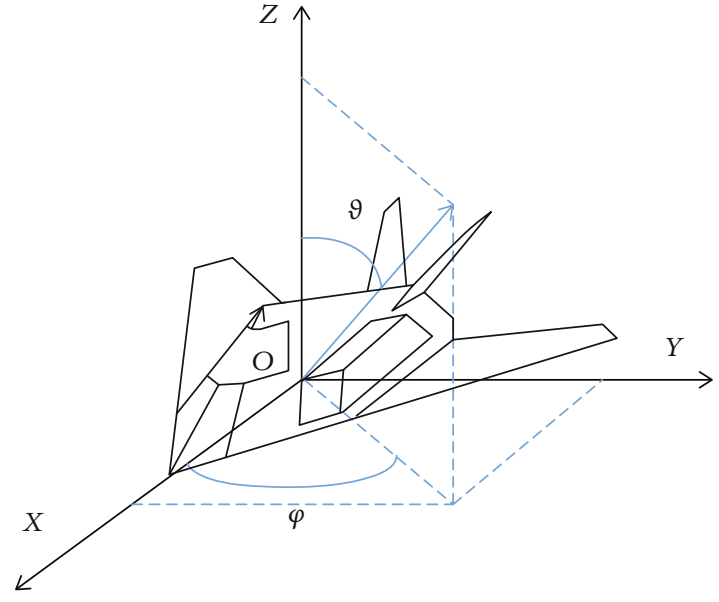

Figure 2: The coordinate system of the target.

process from the radar coordinate system to the target coordinate system is as follows:

$$
\left[\begin{array}{l}
x_{\mathrm{T}}(t) \\
y_{\mathrm{T}}(t) \\
z_{\mathrm{T}}(t)
\end{array}\right]=\left[\begin{array}{lll}
p_{11} & p_{12} & p_{13} \\
p_{21} & p_{22} & p_{23} \\
p_{31} & p_{32} & p_{33}
\end{array}\right] \cdot\left[\begin{array}{c}
x-x_{\mathrm{R}}(t) \\
y-y_{\mathrm{R}}(t) \\
z-z_{\mathrm{R}}(t)
\end{array}\right]=\mathbf{P} \cdot\left[\begin{array}{c}
x-x_{\mathrm{R}}(t) \\
y-y_{\mathrm{R}}(t) \\
z-z_{\mathrm{R}}(t)
\end{array}\right],
$$

where $[x, y, z]$ are the coordinates of a point in the radar coordinate system, $\left[x_{\mathrm{R}}(t), y_{\mathrm{R}}(t), z_{\mathrm{R}}(t)\right]$ are the coordinates of target track in radar coordinate system, $\left[x_{\mathrm{T}}(t), y_{\mathrm{T}}(t), z_{\mathrm{T}}(t)\right]$ are the coordinate of the point $[x, y, z]$ in the target coordinate system, and $\mathbf{P}$ is the transformation matrix, and the elements in the matrix are

$$
\begin{aligned}
& p_{11}=\cos \alpha(t) \cdot \cos \gamma(t)+\sin \alpha(t) \cdot \sin \gamma(t) \cdot \sin \eta(t), \\
& p_{12}=\sin \alpha(t) \cdot \cos \eta(t), \\
& p_{13}=-\cos \alpha(t) \cdot \sin \gamma(t)+\sin \alpha(t) \cdot \cos \gamma(t) \cdot \sin \eta(t), \\
& p_{21}=-\sin \alpha(t) \cdot \cos \gamma(t)+\cos \alpha(t) \cdot \sin \gamma(t) \cdot \sin \eta(t), \\
& p_{22}=\cos \alpha(t) \cdot \cos \eta(t), \\
& p_{23}=\sin \alpha(t) \cdot \sin \gamma(t)+\cos \alpha(t) \cdot \cos \gamma(t) \cdot \sin \eta(t), \\
& p_{31}=\sin \gamma(t) \cdot \cos \eta(t), \\
& p_{32}=-\sin \eta(t), \\
& p_{33}=\cos \gamma(t) \cdot \cos \eta(t),
\end{aligned}
$$

where $\alpha(t), \gamma(t)$, and $\eta(t)$ are yaw angle, pitch angle, and roll angle of the target, respectively. By substituting radar coordinates, then

$$
\left[\begin{array}{c}
x_{\mathrm{T}}^{0}(t) \\
y_{\mathrm{T}}^{0}(t) \\
z_{\mathrm{T}}^{0}(t)
\end{array}\right]=-\mathbf{P} \cdot\left[\begin{array}{c}
x_{\mathrm{R}}(t) \\
y_{\mathrm{R}}(t) \\
z_{\mathrm{R}}(t)
\end{array}\right]
$$




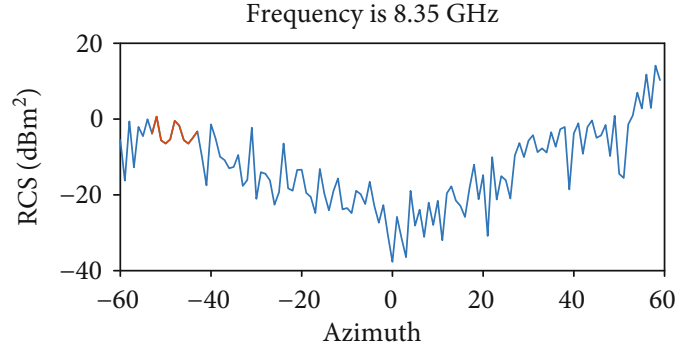

(a)

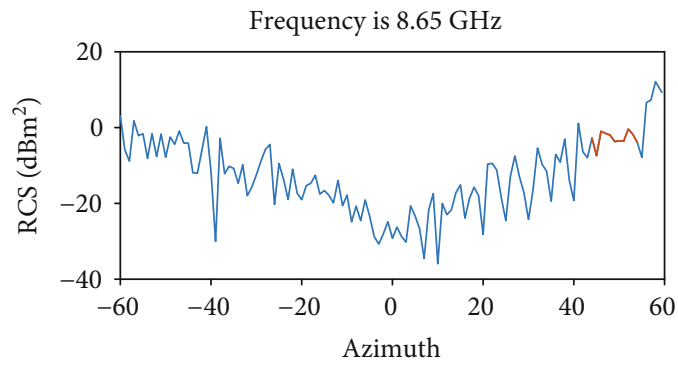

(c)

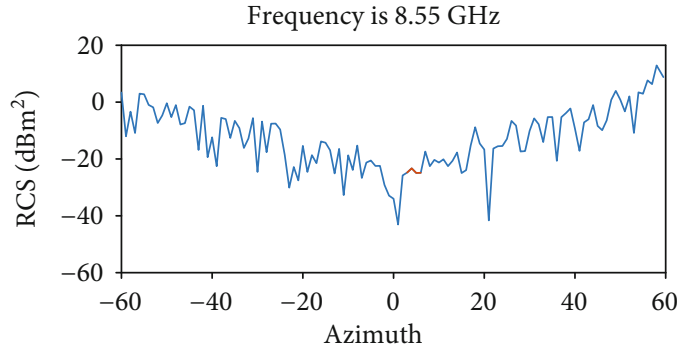

(b)

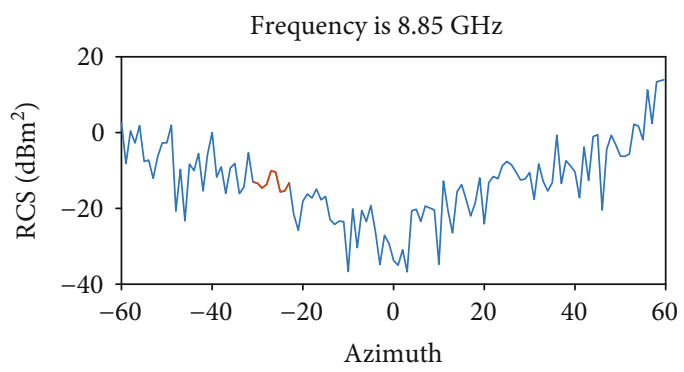

(d)

FIgURE 3: Partition examples constrained by RCS fluctuation loss.

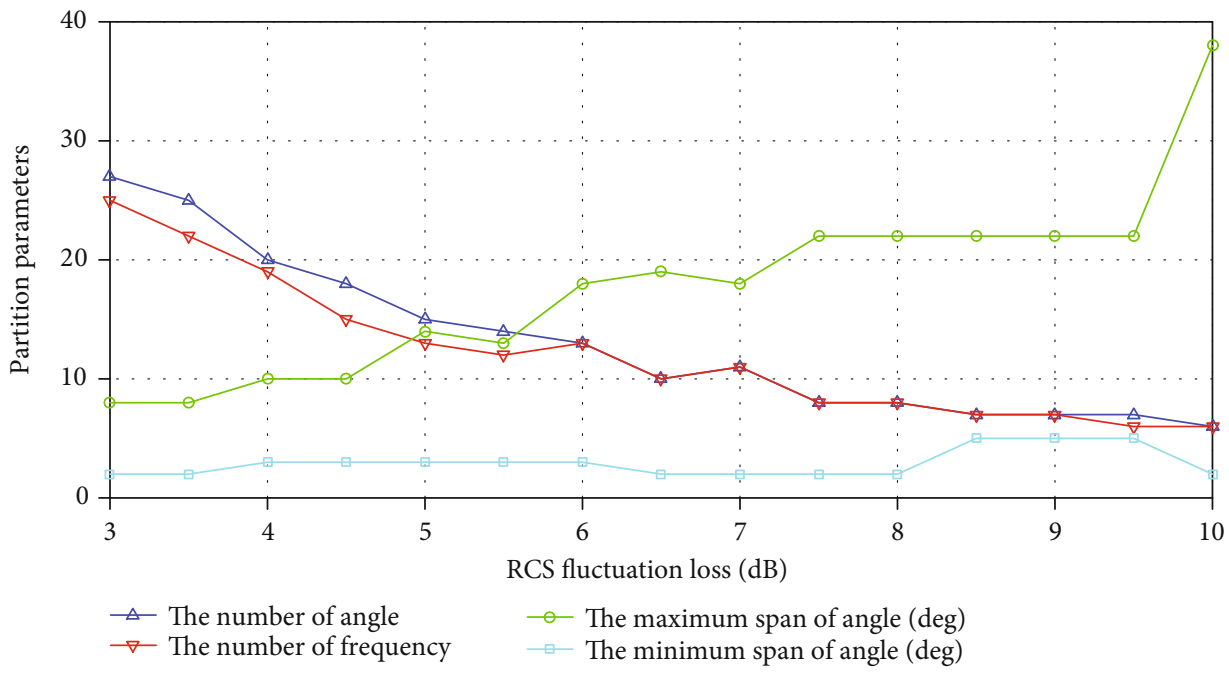

FIgURe 4: Partition parameters constrained by RCS fluctuation loss.

This is the coordinate of the radar in the target coordinate system. From Equation (9), the azimuth angle can be obtained as

$$
\varphi(t)=\arctan \frac{y_{\mathrm{T}}^{0}(t)}{x_{\mathrm{T}}^{0}(t)}
$$

Assume that the azimuth angle of time $k$ predicted at time $k-1$ by target state prediction is $\widehat{\varphi}_{k \mid k-1}$ from Equation (10). The RCS corresponding to the target is obtained from the target RCS database. Azimuth variance $\sigma_{a, k \mid k-1}^{2}$ can be obtained from [12], and the corresponding SNR is estimated as

$$
{\overline{D_{\mathrm{SNR}} k \mid k-1}}=\frac{\overline{D_{\mathrm{SNR} 0}} B^{2} \mu_{\widehat{\varphi}_{k \mid k-1}, f}}{B^{2}+8 \ln (2) \sigma_{a, k \mid k-1}^{2}},
$$

where $B$ represents bandwidth, $\mu_{\widehat{\varphi}_{k \mid k-1}, f}$ is the RCS value corresponding to $\widehat{\varphi}_{k \mid k-1}$ and carrier frequency $f$, and $\overline{D_{\mathrm{SNR} 0 k}}=$ $D_{\text {SNRcal }}\left(T_{k}^{\mathrm{d}} / T_{\text {cal }}^{\mathrm{d}}\right)\left(l_{k \mid k-1} / l_{\text {cal }}\right)^{-4}$. For the dwell time $T_{k}^{\mathrm{d}}$, the calibration parameters $D_{\mathrm{SNR} \text { cal }}, T_{\text {cal }}^{\mathrm{d}}$, and $l_{\text {cal }}$ parameters are set 


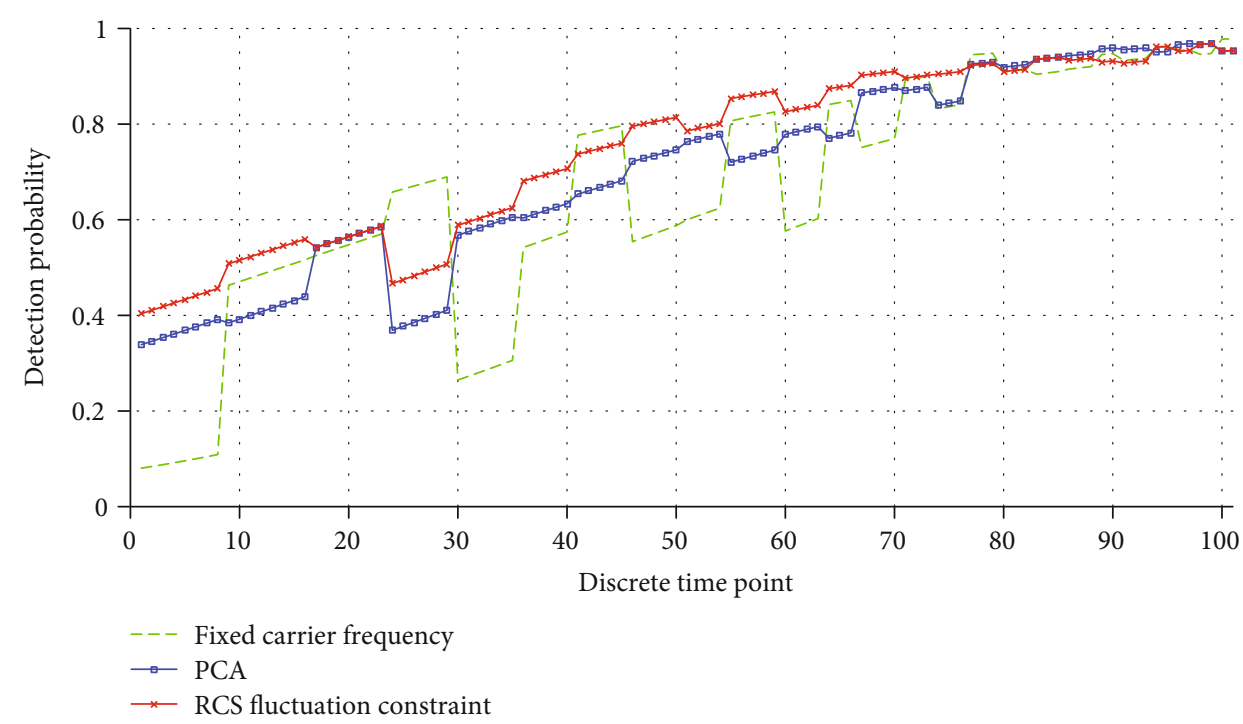

FIGURE 5: Detection probability when classification number is 15 .

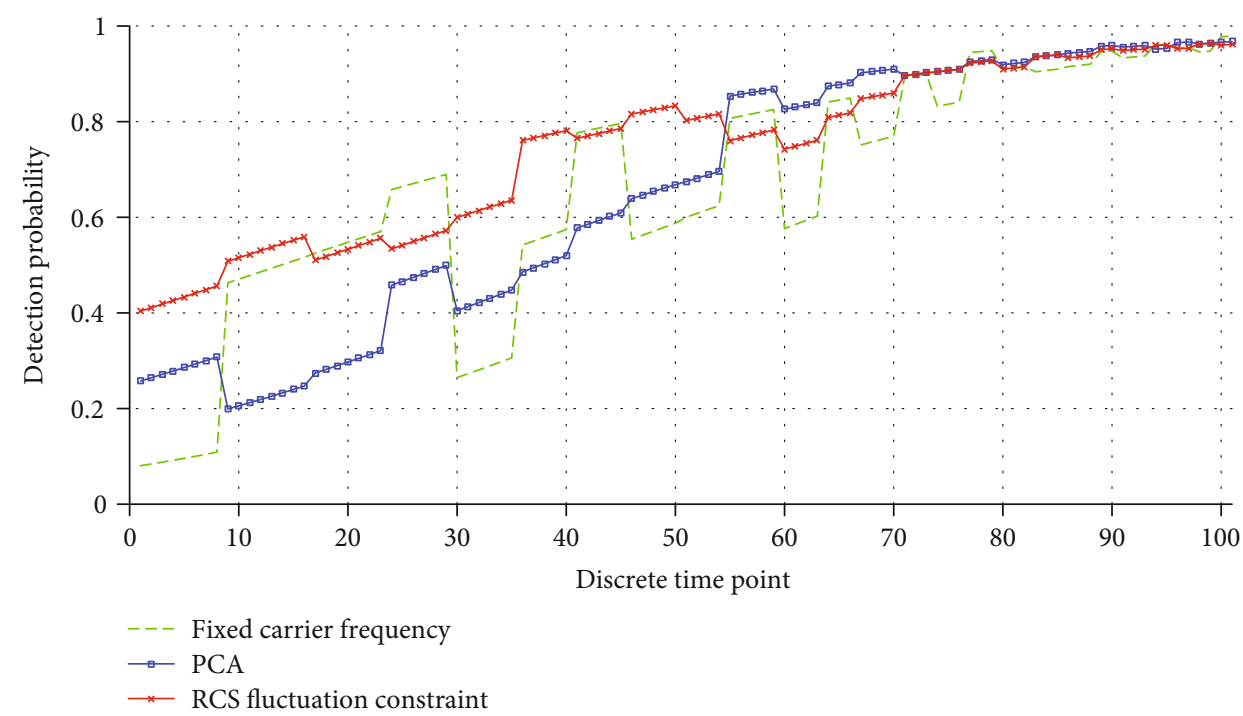

Figure 6: Detection probability when classification number is 20.

to $3 \mathrm{~dB}, 1 \mathrm{~ms}$, and $60 \mathrm{~km}$, respectively; then, the detection probability estimation is

$$
p_{d, k \mid k-1}=p_{\mathrm{fa}}{ }^{1 /\left(1+\overline{D_{\mathrm{SNR} k} k-1}\right)},
$$

where $p_{\mathrm{fa}}$ is false alarm probability. With (11) and (12), the target tracking algorithms based on adaptively sampling interval and dwell time design could be simulated again to further save radar time resource.

4.2. Minimum Dwell Time Design by Interactive Multiple Model Kalman Filter. This paper introduces data set from Section 3 and equations from (10), (11), (12) into the interactive multiple model Kalman filter (IMMKF) algorithm to minimize dwell time. The steps of the algorithm are as follows:

(1) Initialization
Set sampling interval sequence $\Delta \mathbf{T}=\left[\Delta T_{\min }, 2 \Delta T_{\min }, \cdots\right.$, $\left.\Delta T_{\max }\right]$ and dwell time sequence $\tau=\left[\tau_{\min }, 2 \tau_{\min }, \cdots, \tau_{\max }\right]$.

(2) Constructing expected covariance matrix $\mathbf{P}_{\text {des }}$

(3) Estimation predicted error covariance matrix $\mathbf{P}_{\text {pre }}$

(4) Constructing an objective function, for example, let $\mathbf{P}_{\text {des }} \approx \mathbf{P}_{\text {pre }}$

(5) Minimizing radar dwell time

Radar first estimates the dwell time based on the predicted RCS and the target distance to satisfy required detection probability in (12). Since the predicted values of distance and RCS are not exactly accurate, the predicted dwell time could not always meet the requirement. If the current dwell time does not meet the requirement, which might happen for the random variables during tracking process, 


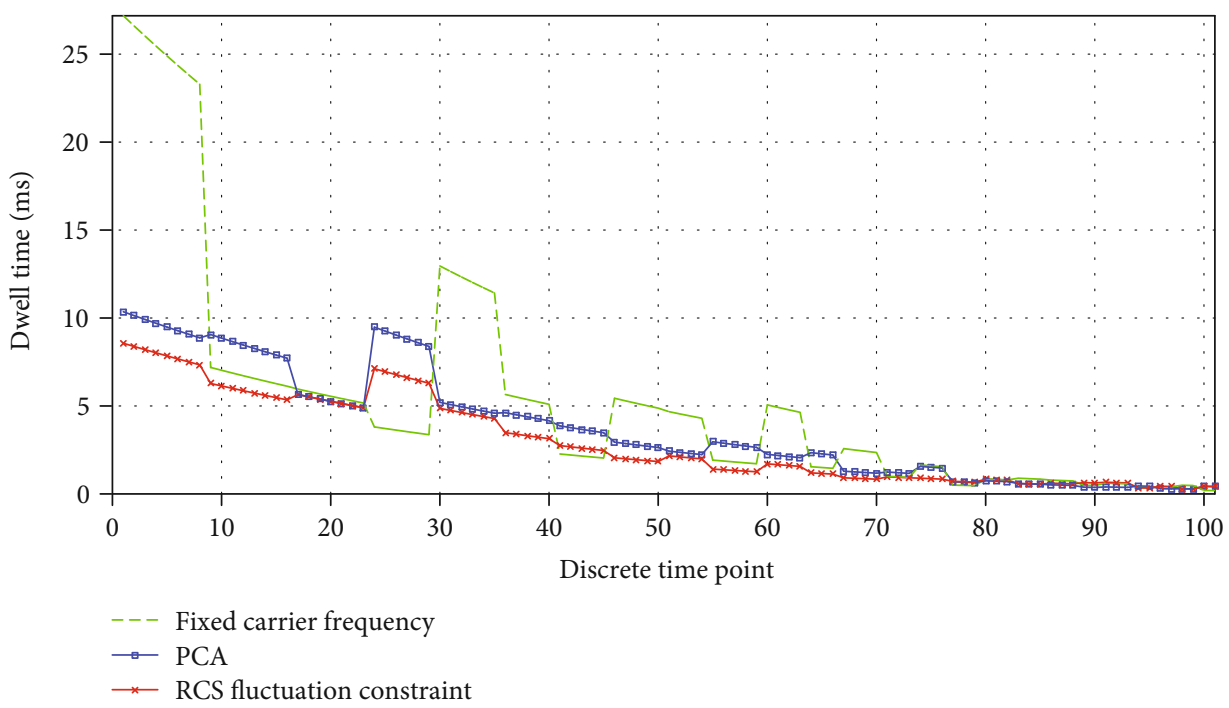

Figure 7: Dwell time when detection probability is 0.8 and classification number is 15 .

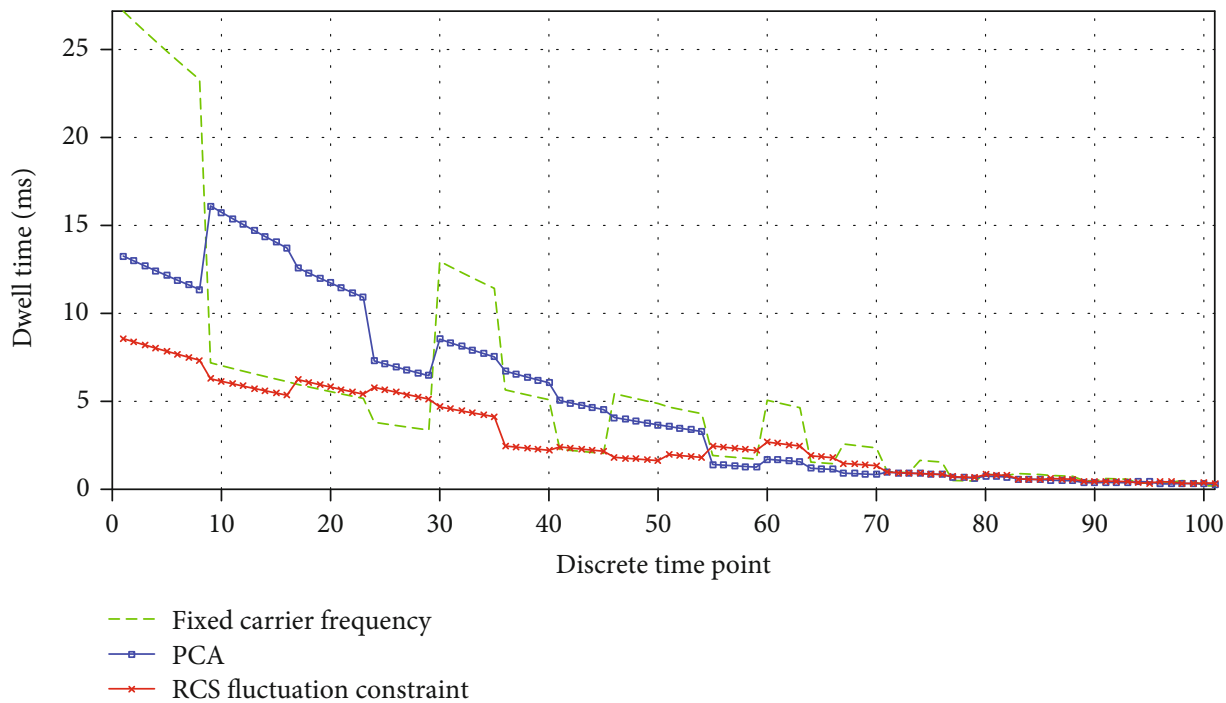

Figure 8: Dwell time when detection probability is 0.8 and classification number is 20 .

such as observation error in (10) and noise, the radar will continue to illuminate the target for $\tau_{\text {min }}$ until the real echo detection probability is equal or greater than the expected detection probability in (12). Besides equations from (10), (11), (12), the detailed IMMKF process is shown in [18].

(6) Status update

\section{Simulations}

This section depicts that the proposed method based on limited RCS fluctuations can be beneficial to the applications in radar target detection and target tracking.

5.1. Simulations to Illustrate Section 3. The RCS data are loaded from simulated RCS database of the target in advance. Figure 3 shows the example of search results of different radar frequencies using the algorithm introduced
TABLE 1: Maneuvering model of targets 2 and 3.

\begin{tabular}{lccc}
\hline $\begin{array}{l}\text { Time } \\
(\mathrm{s})\end{array}$ & $\begin{array}{c}\text { Motion model of } \\
\text { target } 2\end{array}$ & $\begin{array}{c}\text { Time } \\
(\mathrm{s})\end{array}$ & $\begin{array}{c}\text { Motion model of } \\
\text { target } 3\end{array}$ \\
\hline $0-10$ & Uniform & & \\
$11-20$ & Acceleration $\left(10 \mathrm{~m} / \mathrm{s}^{2}\right)$ & & \\
$21-30$ & Acceleration $\left(-20 \mathrm{~m} / \mathrm{s}^{2}\right)$ & $0-10$ & Uniform \\
$31-40$ & Acceleration $\left(20 \mathrm{~m} / \mathrm{s}^{2}\right)$ & $11-30$ & Acceleration $\left(30 \mathrm{~m} / \mathrm{s}^{2}\right)$ \\
$41-60$ & Uniform & $31-100$ & Uniform \\
$61-80$ & Acceleration $\left(20 \mathrm{~m} / \mathrm{s}^{2}\right)$ & & \\
$81-100$ & Acceleration $\left(-40 \mathrm{~m} / \mathrm{s}^{2}\right)$ & & \\
\hline
\end{tabular}

in Section 3. Taking Figure 3(a) as an example, the blue line represents the target RCS at a certain azimuth when carrier frequency is $8.35 \mathrm{GHz}$, and the red line represents a continuous azimuth in which the fluctuation of target RCS is 


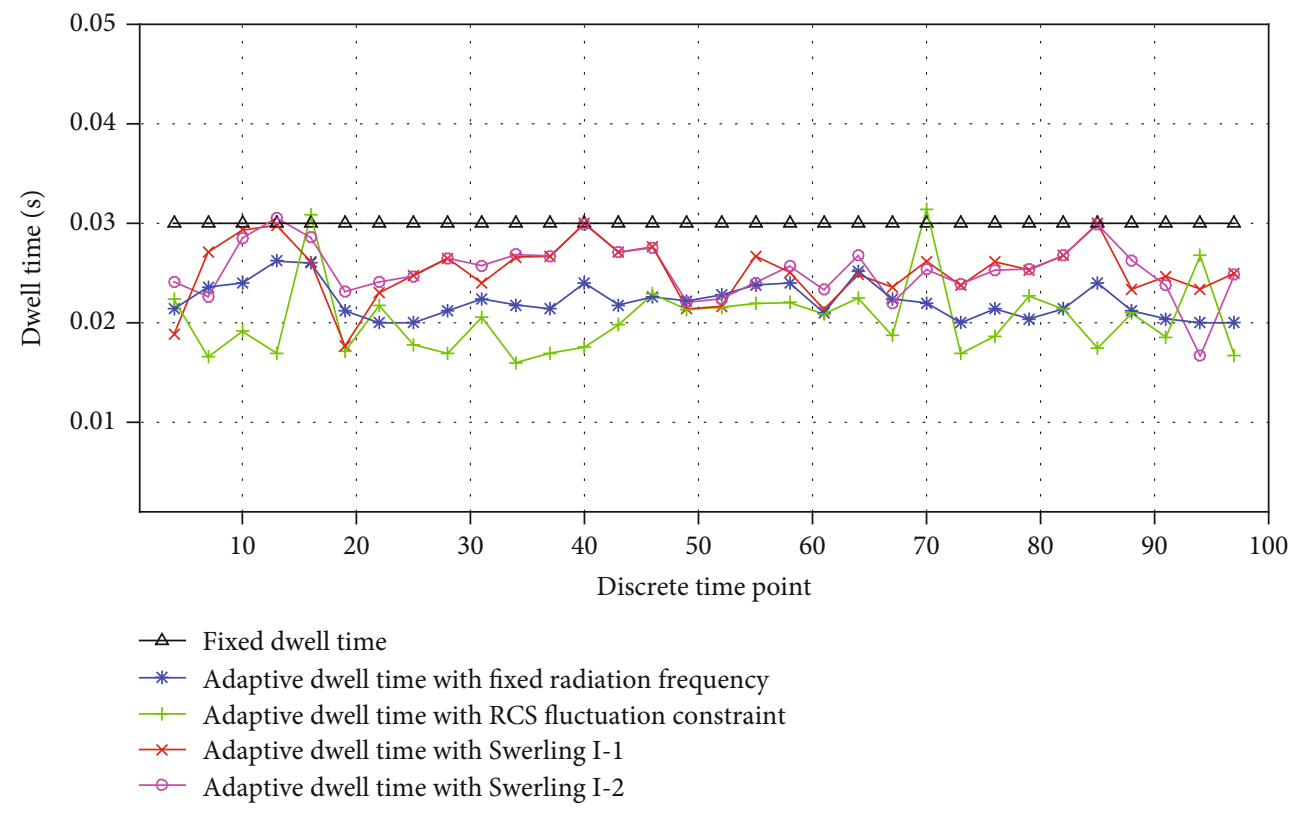

Figure 9: Dwell time comparison on target 1.

constrained to $5 \mathrm{~dB}$. Compared with other different frequencies at the same azimuth, such as (b)-(d) in Figure 3, the red line in (a) depicts that the corresponding RCS values have the average maximum values.

By using the simulated RCS calculated by the HPEM model, Figure 3 also illustrates that the corresponding data are calculated using the algorithm in Section 3, including RCS fluctuation, carrier frequencies, and the span of continuous azimuth. Based on the search results in Figures 3 and 4 shows the partition parameters under different RCS fluctuation constraints, which illustrate that the number of azimuth spans is proportional to the number of required carrier frequencies and is inversely proportional to the tolerate increment of RCS fluctuation.

In addition, Figure 4 also shows that the minimum azimuth span is almost unchanged under different RCS fluctuation constraints. The reason is that there is a dramatic RCS fluctuating, so the average RCS is difficult to be predicted. Meanwhile, the maximum azimuth span will increase as the toleration of RCS fluctuation increases, which indicates that most of the attitude fluctuations of target RCS are in an acceptable range. Therefore, it is possible to use limited RCS fluctuation to save time resources during target tracking process in many cases.

5.2. Simulations to Illustrate Section 4.1. Using the data set simulated in Figure 4, this paper establishes a simple scenario. Supposing that the initial coordinates (unit: $\mathrm{km}$ ) of target 1 is $(80,30,8)$ and this target is moving towards the radar, the velocity and acceleration of the target are $250 \mathrm{~m} / \mathrm{s}$ and $0 \mathrm{~m} / \mathrm{s}^{2}$, respectively; the radar is located at the coordinates $(0$, $0,8)$; the transmitting power is $20 \mathrm{~kW}$; both the antenna gains of the transmitter and receiver are $30 \mathrm{~dB}$; the false alarm probability is $10^{-6}$; the system loss is $5 \mathrm{~dB}$; the receiver noise is $5 \mathrm{~dB}$; and the receiver bandwidth is $1 \mathrm{MHz}$. The detection probability of the target is calculated according to Equation (4).
To illustrate the improvement of detection probability, this paper compares the proposed algorithm with two other methods. One is the fixed carrier frequency method which is used at $8 \mathrm{GHz}$, and the other is the principal component analysis (PCA) method. The PCA method is used to design a carrier frequency which is suitable for each azimuth of a fixed span, so that the target RCS is averaged in that fixed azimuth span. The method proposed in this paper is to design the carrier frequency in an adaptive azimuth span in which the RCS fluctuation is limited. The performance of different methods is shown by detection probability and dwell time from Figures 5-8.

The number of azimuth spans is set to 15 and 20 in order to design the carrier frequency in different azimuth spans within which the RCS fluctuation is limited, and the spans are corresponding to the RCS fluctuation limitation of $5 \mathrm{~dB}$ and $4 \mathrm{~dB}$ in Figure 4. Apparently, this paper takes more prior knowledge of the target RCS calculated by the HPEM model. And the more prior knowledge is utilized, the dwell time will be shorter; thus, the detection probability will be higher.

5.3. Simulations to Illustrate Section 4.2. Besides the simple maneuvering scenario simulated in Section 5.2, this paper takes two other maneuvering trajectories into account to illustrate that the dwell time reduction using the limited RCS fluctuation method. The maneuvering trajectories of target 2 and target 3 are shown in Table 1; they are in the same initial state as target 1.

The relevant parameters in Section 5.2 are set as follows: sampling interval sequence $\Delta \mathbf{T}=5 \mathrm{~s}$ and dwell time sequence $\tau_{\min }=1 \mathrm{~ms},\left|\mathbf{P}_{\text {des }}\right|=240$. And the RCS fluctuation is set to $4 \mathrm{~dB}$.

After 500-time Monte Carlo experiments on the three targets, Figures 9-11 compare the simulation results based on fixed dwell time, adaptive dwell time with fixed radiation frequency, adaptive dwell time with limited RCS fluctuation, 


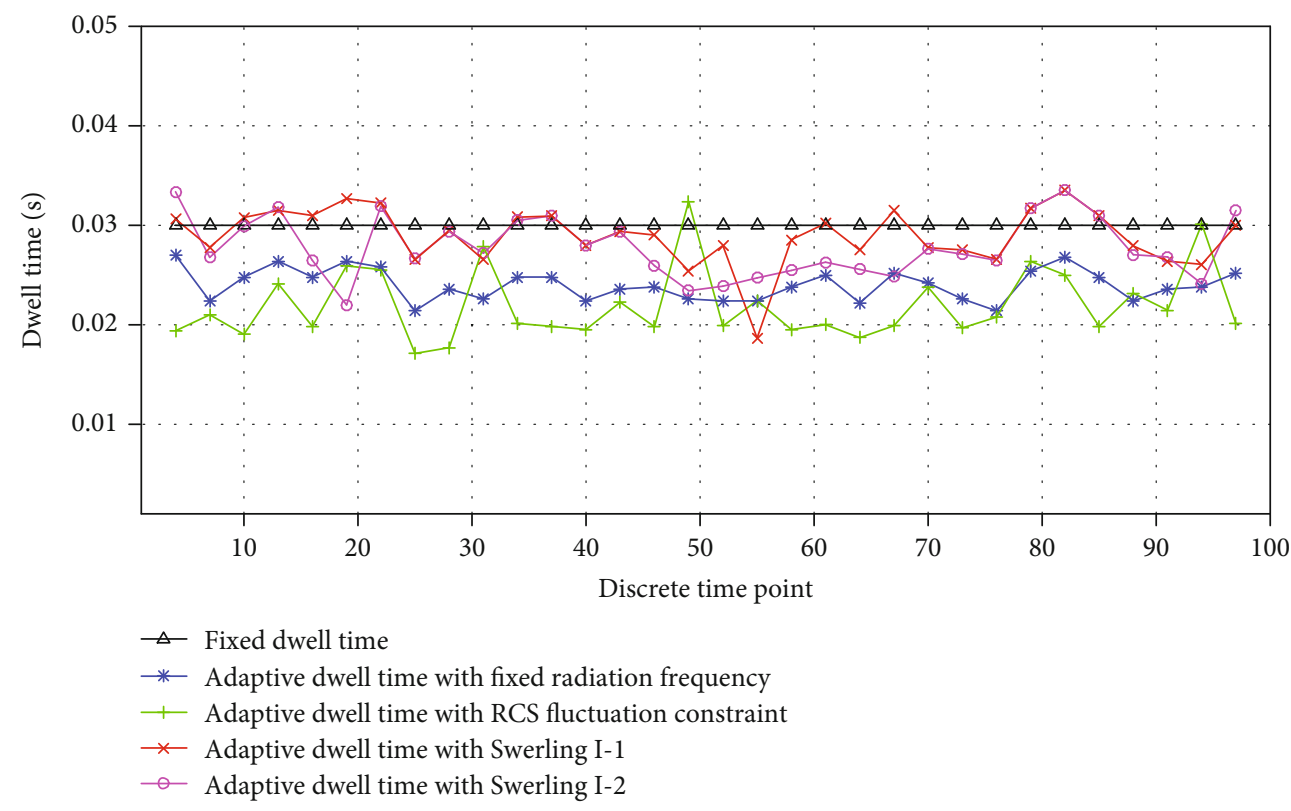

FIGURE 10: Dwell time comparison on target 2.

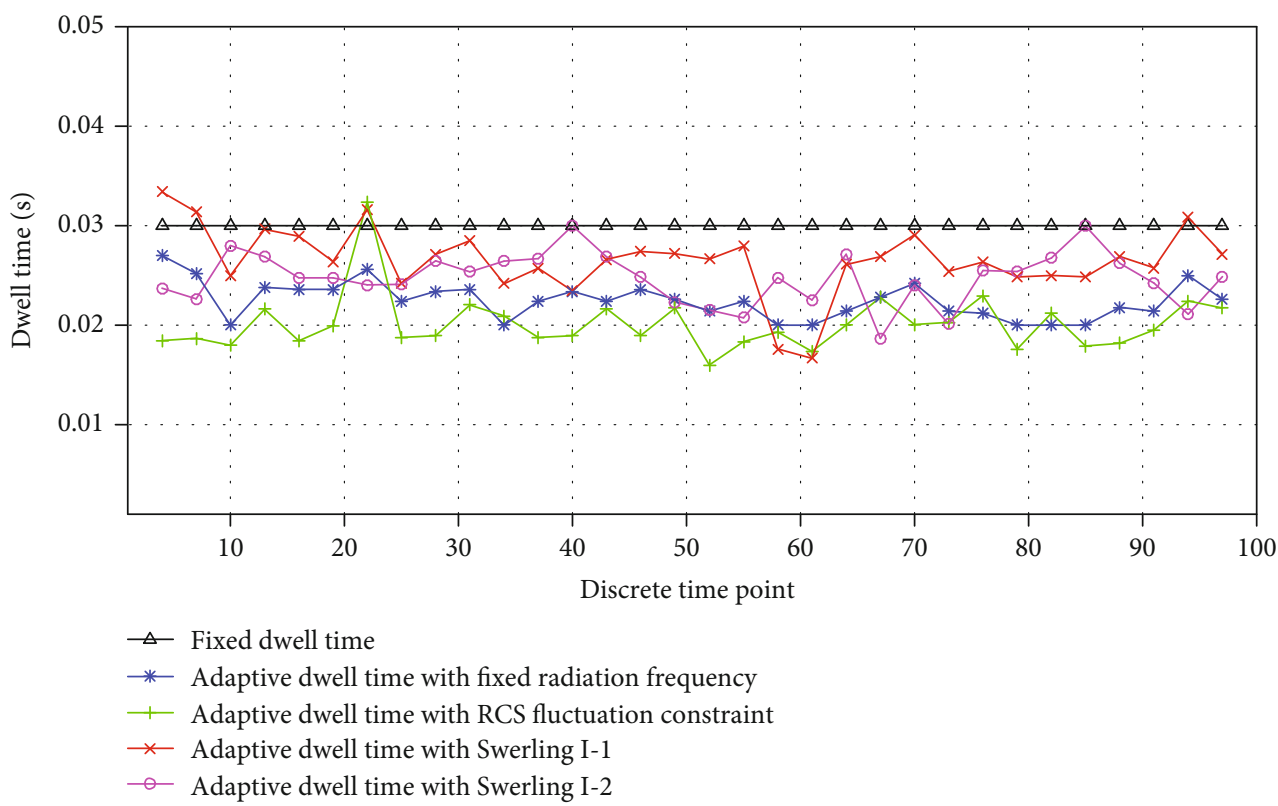

FIGURE 11: Dwell time comparison on target 3.

adaptive dwell time with Swerling I-1, and adaptive dwell time with Swerling I-2. Both Swerling I-1 and Swerling I-2 are Rayleigh models, and their variances are equal to 1 , and the expectation of Swerling I-1 is 0.5 , and the expectation of Swerling I-2 is 1 .

As is shown in Figures 9-11, the dwell time with fixed radiation frequency is less than the fixed dwell time, and the dwell time with limited RCS fluctuation constraint is the shortest. Table 2 shows the statistical average dwell time of target 1 , target 2 , and target 3 .

Compared with the method of fixed dwell time, adaptive dwell time algorithm incorporated with the Swerling model, fixed radiation frequency, and fluctuation constraint require
TABLE 2: Dwell time of target 1 to target 3 (ms).

\begin{tabular}{lccccc}
\hline $\begin{array}{l}\text { Target } \\
\text { index }\end{array}$ & $\begin{array}{c}\text { Fixed } \\
\text { dwell } \\
\text { time }\end{array}$ & $\begin{array}{c}\text { Swerling } \\
\text { I-1 }\end{array}$ & $\begin{array}{c}\text { Swerling } \\
\text { I-2 }\end{array}$ & $\begin{array}{c}\text { Fixed } \\
\text { frequency }\end{array}$ & $\begin{array}{c}\text { Fluctuation } \\
\text { constraint }\end{array}$ \\
\hline 1 & 30 & 26.5 & 26.2 & 22.2 & 19.4 \\
2 & 30 & 27.1 & 27.7 & 24.1 & 19.3 \\
3 & 30 & 26.7 & 26.1 & 24.4 & 20.9 \\
\hline
\end{tabular}

less dwell time. The fixed frequency method can save $7.8 \mathrm{~ms}, 5.9 \mathrm{~ms}$, and $5.6 \mathrm{~ms}$ of dwell time than the fixed dwell time method. Meanwhile, the fluctuation constraint method 
can save $10.6 \mathrm{~ms}, 10.7 \mathrm{~ms}$, and $9.1 \mathrm{~ms}$ of dwell time than the fixed dwell time method. So the method proposed by this paper can save as much as $35.90 \%, 81.35 \%$, and $62.5 \%$ more dwell time than the fixed frequency method during the tracking process.

\section{Conclusions}

This paper proposes a novel method to apply the target RCS calculated by the HPEM model into radar target tracking process, which is effective to save radar time resources. The variable target RCS is considered during the detection probability estimation procedure of the target tracking algorithm, and an adaptive dwell time design is achieved based on the covariance control method. Then, the IMMKF tracking algorithm is improved based on the airborne radar radiation frequency design method. Simulation results illustrate that the proposed method can save average dwell time more effectively during the tracking process compared with other existing methods.

\section{Data Availability}

The data used to support the conclusion of this study are available in Section 5.

\section{Conflicts of Interest}

The authors declare that they have no competing interests.

\section{Authors' Contributions}

All authors equally contributed to this paper.

\section{Acknowledgments}

This research is supported by the Aeronautics Science Foundation of China (Grant No. 2017ZC52036, No. 20172752019), National Natural Science Foundation of China under Grant 61801212, and Natural Science Foundation of Jiangsu Province under Grant BK20180423.

\section{References}

[1] M. Richards, Fundamentals of Radar Signal Processing, Publishing House of Electronics Industry, Beijing, 2017.

[2] X. Song, W. D. Blair, P. Willett, and S. Zhou, "Dominant-plusRayleigh models for RCS: Swerling III/IV versus Rician," IEEE Transactions on Aerospace and Electronic Systems, vol. 49, no. 3, pp. 2058-2064, 2013.

[3] B. Sun, X. Wang, and B. Moran, "Target tracking using range and RCS measurements in a MIMO radar network," in IET International Radar Conference 2013, pp. 1-6, China, April 2013.

[4] J. Liu, N. Fang, F. Bao, and Y. Xie, "A novel dynamic RCS simulation and analysis method considering attitude perturbation," Journal of Electromagnetic Waves Applications, vol. 29, no. 14, pp. 1-18, 2015.

[5] M. Väilä, J. Jylhä, V. Vaisanen et al., "A RCS model of complex targets for radar performance prediction," in 2017 IEEE Radar
Conference (RadarConf), pp. 430-435, Seattle, WA, USA, May 2017.

[6] J. Tan, R. Romero, and D. Jenn, “Target recognition with adaptive waveforms in cognitive radar using practical target RCS responses," in 2018 IEEE Radar Conference (RadarConf18), pp. 606-611, Oklahoma City, OK, USA, 2018.

[7] A. Divyalakshmi, A. Bhuvana, and G. V. Babu, "Analysis of radar cross section and detection," International Journal of Pure and Applied Mathematics, vol. 119, no. 16, pp. 469-477, 2018.

[8] M. R. Bell, "Information theory and radar waveform design," IEEE Transactions on Information Theory, vol. 39, no. 5, pp. 1578-1597, 1993.

[9] J. Liu, K. Wang, and X. Liu, "Waveform design for target detection based on priori characteristics," in 2013 IEEE International Geoscience and Remote Sensing Symposium - IGARSS, pp. 4010-4013, Melbourne, VIC, Australia, July 2013.

[10] C. Shi, F. Wang, M. Sellathurai, J. Zhou, and H. Zhang, "Robust transmission waveform design for distributed multiple-radar systems based on low probability of intercept," ETRI Journal, vol. 38, no. 1, pp. 70-80, 2016.

[11] Z. Zhu, S. Kay, and R. S. Raghavan, "Information-theoretic optimal radar waveform design," IEEE Signal Processing Letters, vol. 24, no. 3, pp. 274-278, 2017.

[12] Z. Zhang and Y. Tian, "A novel resource scheduling method of netted radars based on Markov decision process during target tracking in clutter," EURASIP Journal on Advances in Signal Processing, vol. 2016, Article ID 16, 2016.

[13] J. She, F. Wang, and J. Zhou, "A novel sensor selection and power allocation algorithm for multiple-target tracking in an LPI radar network," Sensors, vol. 16, no. 12, p. 2193, 2016.

[14] J. She, J. Zhou, F. Wang, and H. Li, "LPI optimization framework for radar network based on minimum mean-square error estimation," Entropy, vol. 19, no. 8, p. 397, 2017.

[15] C. Shi, S. Salous, F. Wang, and J. Zhou, "Power allocation for target detection in radar networks based on low probability of intercept: a cooperative game theoretical strategy," Radio Science, vol. 52, no. 8, pp. 1030-1045, 2017.

[16] C. Shi, F. Wang, M. Sellathurai, J. Zhou, and S. Salous, "Power minimization-based robust OFDM radar waveform design for radar and communication systems in coexistence," IEEE Transactions on Signal Processing, vol. 66, no. 5, pp. 13161330, 2018.

[17] C. Shi, F. Wang, S. Salous, and J. Zhou, "Low probability of intercept based multicarrier radar jamming power allocation for joint radar and wireless communications systems," IET Radar, Sonar \& Navigation, vol. 11, no. 5, pp. 802-811, 2017.

[18] F. Wang, S. Yu, C. Shi, and M. Sellathurai, "LPI time-based TMS against high-sensitivity ESM," IET Radar, Sonar \& Navigation, vol. 12, no. 12, pp. 1509-1516, 2018.

[19] M. Skolnik, Radar Handbook, Publishing House of Electronics Industry, Beijing, 3rd edition, 2010. 